\title{
Effet Du Niveau Protéique Et Du Traitement Des Graines De Pois D'angole (Cajanus Cajan (L.) Huth, 1893) Sur La Croissance Du Poulet Local (Gallus Gallus Domesticus, Linnaeus 1758) En Côte d'Ivoire
}

Traore Beh, PhD

Laboratoire de Biologie et Cytologie Animale, UFR Sciences de la nature,

Université Nangui Abrogoua, Abidjan Côte d'Ivoire

Orsot Bosso M'bolo Marcel Roland, MA

Komara Moussa, PhD

Ouattara Siaka, PhD

Dofara Soro, PhD

Université Nangui Abrogoua, Abidjan Côte d'Ivoire

Doi: 10.19044/esj.2018.v14n36p452 URL:http://dx.doi.org/10.19044/esj.2018.v14n36p452

\begin{abstract}
This paper focuses on evaluating the effect of heat treatment of pigeon pea grains as a source of protein and protein level on local chicken growth. To conduct this study, 135 locally grown one-day-old chicks of roughly equal weight were randomized into 9 groups of equal size. Each group was subdivided into five lots of 3 subjects that were housed in a boxing. Then, three types of treatment (roasting, steaming and drying) were applied to the grains. For each type of treatment, three different protein level foods were developed. Each batch of chicks was fed with a given food. The results showed significant interactions between the protein level and the nature of the heat treatment on the different parameters (PTT $<\alpha$, PNP $<\alpha$ and PTT $*$ NP $<\alpha$ ). Food consumption decreased significantly with protein level and was lower for CG and CV foods. The average daily gain and the feed index of batches fed with CG and CV foods were the best. Depending on the protein level, those titrated at $13 \%$ achieved the best growth and feed efficiency. Despite a lack of significant effect of the protein level on protein digestibility, there was a slight drop in protein at a dose of $16 \%$. The nature of the treatment, for its part, significantly influenced the digestibility of proteins.
\end{abstract}

Keywords: Cajanus cajan, croissance, niveau protéique, poulet, traitement thermique 


\section{Resume}

Cette étude a pour objectif d'évaluer l'effet du traitement thermique des graines de pois d'angole comme source de protéines et du niveau protéique des aliments sur la croissance du poulet local. Pour y parvenir, 135 poussins agés d'un jour, de race locale et de poids sensiblement égal ont été repartis par tirage aléatoire en 9 groupes d'égal effectif. Chaque groupe a été subdivisé en cinq lots de 3 sujets qui ont été logés dans un boxe. Puis, trois types de traitement (torréfaction, cuisson à la vapeur et séchage) ont été appliqués aux graines. Ensuite, pour chaque type de traitement, trois aliments de niveau protéique différent ont été élaborés. Chaque lot de poussins a été nourri avec un aliment donné. Les résultats ont montré des interactions significatives entre le taux de protéine et la nature du traitement thermique sur les différents paramètres $\left(\mathrm{P}_{\mathrm{TT}}<\alpha, \mathrm{P}_{\mathrm{NP}}<\alpha\right.$ et $\left.\mathrm{P}_{\mathrm{TT}} * \mathrm{NP}<\alpha\right)$. La consommation alimentaire a baissé de manière significative avec le niveau protéique et était plus faible pour les aliments avec les graines de cajanus torréfiées $(\mathrm{CG})$ et cuites à la vapeur $(\mathrm{CV})$. Le gain de poids moyen quotidien et l'indice de consommation des lots nourris avec les aliments $\mathrm{CG}$ et $\mathrm{CV}$ étaient les meilleurs. Selon le taux de protéine, ceux titrés à $13 \%$ ont obtenu les meilleures croissance et efficacité alimentaire. Les mortalités étaient plus importantes pour les poulets nourris avec les rations à $16 \%$ de protéines. Les rations à $13 \%$ de protéines constituées de graines $\mathrm{CG}$ et CV se sont révélées les meilleures.

Mots-clés: Phytodiversité, végétation, groupements végétaux, Côte d'Ivoire

\section{Introduction}

Le poulet local est généralement considéré comme l'oiseau le plus domestiqué dans les zones rurales. Il représente $70 \%$ des effectifs de volailles et provient du secteur familial. Son élevage occupe une place socioéconomique particulière au sein des populations et est pratiqué tant en milieu rural qu'urbain (Saliou \& Danho, 2008). L'aviculture familiale représente pour ces populations un moyen d'épargne et une source de revenus (FAO, 2004). Ainsi, elle permet de lutter contre la pauvreté et l'insécurité alimentaire des populations rurales en particulier chez les femmes et enfants qui en ont la garde (Gueye, 2000 ; Missohou et al., 2002). Le mode d'élevage variant selon les moyens disponibles (Aklobessi et al., 1992) est essentiellement de type traditionnel caractérisé par une très faible productivité (Guèye, 1998). Les principales contraintes de ce système, qui sont d'ordre pathologique et alimentaire, entrainent une faible productivité numérique et pondérale. En effet, pour se nourrir, les poulets divaguent à longueur de journée à travers les concessions et les champs. Quelques éleveurs apportent des compléments sous forme de grains de maïs, d'insectes (termites) et des restes de nourriture. 
Toutefois, la disponibilité de ces aliments en quantités suffisantes n'est pas toujours assurée. De plus, les rations constituées ne sont pas équilibrées. Pourtant, des travaux (Sonaiya, 1995 ; Sarkar \& Bell, 2006) ont montré qu'une complémentation alimentaire équilibrée aide à maximiser les performances de ponte, de croissance et la productivité des poules locales. Tout ceci démontre que l'alimentation est un facteur déterminant pour la productivité du poulet local (Akouango et al., 2004). Par ailleurs, le coût élevé des aliments commerciaux formulés pour des animaux à hautes performances génétiques, limite leur utilisation en milieu rural à cause du coût élevé des intrants. Il devient donc impératif de réduire le coût des aliments par la recherche et la valorisation de matières premières locales, disponibles et bon marché puis de la réalisation de ration adaptée aux besoins des poulets locaux.

Ainsi, plusieurs travaux ont été menés pour évaluer l'emploi des légumineuses à graines dans l'alimentation des volailles dont ceux de Ouattara et al. (2014) sur le niébé et de Okandza et al. (2017) sur la féverole. Parmi ces légumineuses, figure en bonne place le pois d'angole (Cajanus cajan). En effet, ces graines très peu utilisées dans l'alimentation des hommes sont disponibles en grande quantité dans les principales zones de production où la plante est utilisée pour la fertilisation et la restauration des sols. Elles sont relativement énergétiques, riches en protéines (Amaefule \& Obioha, 2005), appréciées de la volaille et peuvent être utilisées dans l'alimentation des poulets locaux (Niyonkuru, 2002). Cependant, les graines de Cajanus cajan tout comme les autres légumineuses renferment des facteurs antinutritionnels dont l'effet peut être réduit par divers traitements (cuisson, torréfaction, séchage, trempage, germination, fermentation) (Grimaud, 1988 ; Chrysostome et al., 1998 ; Ayssiwede et al., 2012 ; Ouedraogo et al., 2015). L'objectif général de ce travail est de rechercher une voie alternative d'amélioration de l'alimentation et de la productivité des poulets locaux. De façon spécifique, il s'agira d'évaluer l'effet des traitements thermiques des graines du pois d'angole et leur niveau d'incorporation pour une croissance optimale des poulets locaux.

\section{Matériel et méthodes}

Cette étude s'est étendue sur une période de 109 jours. Elle a été réalisée sur le site de la ferme Carrière (ferme experimentale de l'université Nangui Abrogoua située à Céchi).

\section{Zone de l'étude}

Le présent travail a été réalisé dans la localité de Céchi, une souspréfecture du département d'Agboville dans la région de l'Agnéby Tiassa. Le climat de Céchi est de type tropical humide caractérisé par deux saisons des pluies et deux saisons sèches. La grande saison sèche dure de décembre à avril 
et la grande saison humide de mai à juillet. La petite saison sèche dure d'août à septembre et la petite saison humide d'octobre à novembre (Picq et al., 1992 ; Ouédraogo, 2001). Les sols sont sablonneux et argileux.

Dans cette localité, l'élevage de poulet local est pratiqué par tous les ménages et le système d'élevage est de type extensif (Alla-Sombo \& Fousseni, 2005).

\section{Animaux expérimentaux}

L'essai a porté sur 135 poussins agés d'un jour, d'un poids moyen de $25 \mathrm{~g}$. Ces poussins ont été obtenus à l'issu de l'incubation d'œufs de poules de race locale collectés dans la zone d'étude. Après éclosion, chaque poussin a subi un examen physique pour s'assurer de son aptitude physique Cet examen a consisté à vérifier la vivacité du poussin, la qualité du duvet, les aplombs, l'absence de malformations ou de paralysies et une bonne cicatrisation de 1'ombilic. Les poussins ont été repartis de façon aléatoire en 9 groupes d'égal effectif. Puis, chaque groupe a été subdivisé en 5 lots de trois individus. Les lots d'animaux ont été logés dans des boxes en raison d'un lot par boxe.

\section{Traitement des graines de Cajanus cajan}

Les graines après la récolte, ont été triées pour retirer les mauvaises. Puis, elles ont été analysées pour déterminer leur composition chimique (Protéines brutes, cendres brutes et matière organique) avant de subir trois traitements thermiques. Il s'agit de la torréfaction, de la cuisson à la vapeur et du séchage.

La torréfaction a consisté à mettre $5 \mathrm{~kg}$ de graines de $C$. cajan dans une casserole posée sur le feu. Ensuite, les graines ont été régulièrement remuées durant 30 minutes. Après la torréfaction, les graines ont été refroidies à l'air libre pendant une heure. L'opération a été reprise pour avoir la quantité necessaire.

Pour la cuisson à la vapeur, une passoire a été posée au-dessus d'une marmite contenant 31 d'eau. Le tout a été placé sur le feu. Dès ébullition de l'eau, $5 \mathrm{~kg}$ de graines de pois d'angole ont été mis dans la passoire. Trente minute après, les graines ont été égouttées puis étalées à l'ombre pour un séchage de deux heures.

Pour le dernier traitement, les graines ont été étalées sur une bâche puis exposées au soleil durant deux jours. À intervalle de temps régulier, elles ont été retournées.

Après les différents traitements, les graines ont été concassées au moulin artisanal avant leur incorporation dans les différentes rations. 


\section{Formulation et distribution des rations expérimentales}

À la suite de ces traitements, les graines concassées de $C$. cajan ont été mélangées au maïs concassé de manière à obtenir trois niveaux protéiques différents par traitement (Tableau I). Les valeurs bromatologiques des matières premières ont servi de base pour la formulation des rations. Ces rations ont été élaborées par un programme à l'aide du tableur Excel (2016) de sorte à obtenir 10,13 et $16 \%$ de protéines. Après l'élaboration, des compléments minéraux à base de coquille d'escargot ont été ajoutés à raison de $2 \%$. Ensuite, les teneurs en protéines brutes, cendres brutes et en matière organique ont été évaluées. Le Tableau I présente les différentes rations ainsi que leurs compositions chimiques, le taux d'incorporation des différents ingrédients et la distribution des différents aliments. Ainsi, un type d'aliment particulier a été attribué à chaque groupe (Tableau I).

Tableau I. Traitement, différentes rations, taux d'incorporation des différents ingrédients et composition chimique des aliments expérimentaux

\begin{tabular}{|c|c|c|c|c|c|c|c|}
\hline \multirow{2}{*}{$\begin{array}{l}\text { Traitements } \\
\text { thermiques } \\
\text { appliqués }\end{array}$} & \multirow[t]{2}{*}{$\begin{array}{c}\text { Groupes } \\
\text { d'animaux }\end{array}$} & \multirow[t]{2}{*}{$\begin{array}{l}\text { Code des } \\
\text { aliments }\end{array}$} & \multicolumn{2}{|c|}{$\begin{array}{c}\text { Taux } \\
\text { d'incorporation (\%) }\end{array}$} & \multicolumn{3}{|c|}{ Teneur en \% MS } \\
\hline & & & $\begin{array}{l}\text { Cajanus } \\
\text { cajan }\end{array}$ & maïs & Prot. & $\mathrm{CB}$ & $\mathrm{MO}$ \\
\hline \multirow[t]{3}{*}{ Séchage } & Goupe 1 & $\mathrm{CS}_{10}$ & 10,69 & 83,5 & 10,22 & 1,59 & 98,41 \\
\hline & Goupe 2 & $\mathrm{CS}_{31}$ & 31,77 & 68,22 & 13,54 & 2,25 & 97,75 \\
\hline & Goupe 3 & $\mathrm{CS}_{47}$ & 47,95 & 52,04 & 16,22 & 2,77 & 97,23 \\
\hline \multirow{3}{*}{$\begin{array}{l}\text { Cuisson à la } \\
\text { vapeur }\end{array}$} & Goupe 4 & $\mathrm{CV}_{10}$ & 10,69 & 83,5 & 10,22 & 1,59 & 98,41 \\
\hline & Goupe 5 & $\mathrm{CV}_{31}$ & 31,77 & 68,22 & 13,54 & 2,25 & 97,75 \\
\hline & Goupe 6 & $\mathrm{CV}_{47}$ & 47,95 & 52,04 & 16,22 & 2,77 & 97,23 \\
\hline \multirow[t]{3}{*}{ Torréfaction } & Goupe 7 & $\mathrm{CG}_{10}$ & 10,69 & 83,5 & 10,22 & 1,59 & 98,41 \\
\hline & Goupe 8 & $\mathrm{CG}_{31}$ & 31,77 & 68,22 & 13,54 & 2,25 & 97,75 \\
\hline & Goupe 9 & $\mathrm{CG}_{47}$ & 47,95 & 52,04 & 16,22 & 2,77 & 97,23 \\
\hline
\end{tabular}

CS : Cajanus séché ; CV : Cajanus cuit à la vapeur ; CG : Cajanus torréfié

Prot $=$ protéine $; \mathrm{CB}=$ Cendres brutes $; \mathrm{MO}=$ Matière organique

\section{Conduite de l'expérimentation}

Avant l'installation des poussins, les différents boxes ont été désinfectés puis, une litière de copeau de bois a été mise en place. Les poussins ont été chauffés jusqu'à 21 jours d'âge. L'éclairage a été permanent durant les 6 semaines d'expérimentation.

Les aliments ont été distribués ad libitum deux fois par jour, les matins à 06 heures et les soirs à 17 heures de un jour à 6 semaines d'âge. L'eau a également été distribuée à volonté. Au cours de cette expérimentation, les poussins ont été vaccinés, déparasités et ont reçu un anticoccidien (Tableau II). Un complexe vitaminé a été apporté en eau de boisson à intervalle de 15 jours. Il fût dosé à $5 \mathrm{mg}$ pour $10 \mathrm{l}$ d'eau. 
Tableau II. Prophylaxie médicale suivie au cours de l'expérimentation

\begin{tabular}{|c|c|c|c|}
\hline Âge (jour) & Traitements & Produits utilisés & Dosage \\
\hline 6 & $\begin{array}{l}\text { Vaccin contre la pseudo-peste aviaire } \\
\text { et la bronchite infectieuse }\end{array}$ & HB1 - H120 & 1 dose / oiseau \\
\hline 12 & Vaccin contre la bursite infectieuse & Gumboral & 1 dose / oiseau \\
\hline 15 & Vitamine & Amin'total & 5 mg / 101 \\
\hline 20 & $\begin{array}{l}\text { Rappel vaccin contre la pseudo-peste } \\
\text { aviaire }\end{array}$ & La SOTA & 1 dose / oiseau \\
\hline 30 & Anticoccidien et vitamine & $\begin{array}{l}\text { Amprocox - } \\
\text { Amin'total }\end{array}$ & $5 \mathrm{mg} / 101$ \\
\hline 32 & Déparasitage & Levozan & $5 \mathrm{mg} / 51$ \\
\hline 35 & $\begin{array}{l}\text { Rappel vaccin contre la bronchite } \\
\text { infectieuse }\end{array}$ & H120 & 1 dose / oiseau \\
\hline
\end{tabular}

\section{Étude de la digestibilité des aliments expérimentaux}

Afin d'étudier la digestibilité, 5 poulets de 5 semaines d'âge de chaque lot ont été logés individuellement dans des cages de digestibilité. L'expérimentation a duré 10 jours, au cours desquelles les quantités d'aliment distribuées et refusées sont pesées quotidiennement de même que la quantité de fiente excrétée. Ces différents échantillons ont été par la suite séchés pour déterminer leur teneur en matière sèche. À la fin de l'expérimentation, les fientes obtenues ont été mélangées par lots en vue de déterminer leur teneur en protéines brutes, cendres brutes et en matière organique selon la méthode AFNOR (1993). La digestibilité de ces différents éléments et de la matière sèche a été déterminée à l'aide du coefficient d'utilisation digestif apparent comme indiqué par la formule ci-après.

\section{Mesures des paramètres}

$$
\text { CUD apparent }=\frac{\text { Ingéré }- \text { Exctrété }}{\text { Ingéré }} \text { X } 100
$$

La consommation d'aliment, le poids des animaux, le gain de poidf vif moyen quotidien (GMQ), l'indice de consommation et le taux de mortalité ont été déterminés. La consommation alimentaire quotidienne des poulets a été obtenue par différence entre la quantité servie et le refus. Le poids vif des animaux a été déterminé par pesée hebdomadaire. Chaque sujet a été pesé individuellement. Le gain de poids moyen quotidien, l'indice de consommation et le taux de mortalité ont été calculés par semaine selon les formules suivantes :

$$
\begin{gathered}
\mathrm{IC}=\frac{\text { Quantité moyenne d'aliment consommé pendant une période }(\mathrm{g})}{\text { Gain de poids moyen durant la période }} \\
\mathrm{GMQ}=\frac{\text { Gain de poids pendant une période }(\mathrm{g})}{\text { Durée de la période }(\mathrm{jrs})} \\
\mathrm{TM}=\frac{\text { Nombre de mortalité }}{\text { Éffectif initial }} \times 100
\end{gathered}
$$




\section{Analyse statistique}

Les différentes données obtenues ont été enregistrées et traitées dans le tableur de Microsoft Excel 2016. La consommation alimentaire, le GMQ et l'indice de consommation ont été soumis au test d'analyse de la variance (ANOVA) à deux facteurs au seuil de $5 \%$ à l'aide du logiciel Statistica 7.0 et complété par le test HDS de Tukey lorsque le test d'analyse de la variance a montré une différence significative. L'analyse des résultats du coefficient de digestibilité a été faite par l'analyse des variances à un facteur puis au test HDS de Tukey. Quant au taux de mortalité, il subit une analyse descriptive.

\section{RÉSULTATS}

\section{Effet du traitement thermique et du niveau de protéine sur la consommation alimentaire}

Comme le montre le Tableau III, la consommation alimentaire diminue avec l'augmentation du niveau de protéine. Les rations titrées à $10 \%$ de protéine, quel que soit le traitement thermique appliqué recensent les moyennes de consommation les plus élevées. Les rations avec les graines de Cajanus cajan séchées ont été significativement plus consommées que les rations avec les graines de $C$. Cajanus cuites à la vapeur ou torréfiées. De façon générale, il est constaté que le taux de protéine et le traitement thermique de même que leur interaction influencent la consommation alimentaire.

Tableau III. Consommation alimentaire en fonction du traitement thermique et du niveau protéique

\begin{tabular}{cccccccccc}
\hline \multirow{2}{*}{$\begin{array}{c}\text { Age } \\
\text { (semaine) }\end{array}$} & \multicolumn{3}{c}{ Traitements } & \multicolumn{1}{c}{ Niveaux protéiques } & \multicolumn{3}{c}{ P value } \\
\cline { 2 - 10 } & CG & \multicolumn{1}{c}{ CV } & CS & 10 & 13 & 16 & TT & NP & TT*NP \\
\hline 1 & $18,88^{\mathrm{a}}$ & $22,12^{\mathrm{a}}$ & $19,76^{\mathrm{a}}$ & $25,86^{\mathrm{b}}$ & $16,58^{\mathrm{a}}$ & $18,33^{\mathrm{a}}$ & 0,551 & $0,005^{*}$ & 0,803 \\
& $\pm 2,08$ & $\pm 2,21$ & $\pm 2,04$ & $\pm 1,99$ & $\pm 2,03$ & $\pm 2,30$ & & & \\
2 & $20,09^{\mathrm{a}}$ & $25,39^{\mathrm{ab}}$ & $26,33^{\mathrm{b}}$ & $28,38^{\mathrm{b}}$ & $19,74^{\mathrm{ab}}$ & $23,68^{\mathrm{a}}$ & $0,001^{*}$ & $0,016^{*}$ & 0,329 \\
& $\pm 1,57$ & $\pm 1,75$ & $\pm 1,54$ & $\pm 1,50$ & $\pm 1,62$ & $\pm 1,74$ & & & \\
3 & $24,04^{\mathrm{a}}$ & $24,24^{\mathrm{a}}$ & $27,63^{\mathrm{a}}$ & $26,11^{\mathrm{a}}$ & $21,88^{\mathrm{a}}$ & $27,92^{\mathrm{a}}$ & 0,390 & 0,141 & 0,195 \\
& $\pm 2,05$ & $\pm 2,289$ & $\pm 2,01$ & $\pm 1,96$ & $\pm 2,12$ & $\pm 2,27$ & & & \\
4 & $21,97^{\mathrm{a}}$ & $26,84^{\mathrm{a}}$ & $28,56^{\mathrm{a}}$ & $37,73^{\mathrm{b}}$ & $22,69^{\mathrm{a}}$ & $16,95^{\mathrm{a}}$ & 0,005 & $0,000^{*}$ & $0,000^{*}$ \\
& $\pm 1,40$ & $\pm 1,44$ & $\pm 1,38$ & $\pm 1,24$ & $\pm 1,442$ & $\pm 1,53$ & & & \\
5 & $28,06^{\mathrm{a}}$ & $28,67^{\mathrm{a}}$ & $37,94^{\mathrm{b}}$ & $43,70^{\mathrm{b}}$ & $28,46^{\mathrm{a}}$ & $22,51^{\mathrm{a}}$ & $0,002^{*}$ & $0,000^{*}$ & $0,005^{*}$ \\
& $\pm 1,77$ & $\pm 1,81$ & $\pm 2,16$ & $\pm 1,45$ & $\pm 1,81$ & $\pm 2,38$ & & & \\
& $32,70^{\mathrm{a}}$ & $30,08^{\mathrm{a}}$ & & $49,12^{\mathrm{b}}$ & $30,92^{\mathrm{a}}$ & $21,78^{\mathrm{a}}$ & 0,232 & $0,000^{*}$ & $0,001^{*}$ \\
& $\pm 1,51$ & $\pm 1,54$ & & $\pm 1,24$ & $\pm 1,72$ & $\pm 4,02$ & & & \\
\hline Moyenne & $24,31^{\mathrm{a}}$ & $25,43^{\mathrm{a}}$ & $29,12^{\mathrm{b}}$ & $35,15^{\mathrm{b}}$ & $21,91^{\mathrm{a}}$ & $21,79^{\mathrm{a}}$ & $0,006^{*}$ & $0,000^{*}$ & $0,006^{*}$ \\
& $\pm 1,07$ & $\pm 1,13$ & $\pm 1,05$ & $\pm 1,02$ & $\pm 1,04$ & $\pm 1,18$ & & & \\
\hline
\end{tabular}

Moyenne \pm écart-type

*indique les différences significatives des effets (traitement et niveau protéique)

Les valeurs suivies de la même lettre pour une même ligne et par facteur ne sont significativement différentes au seuil de $5 \%$. 


\section{Effet du traitement thermique et du niveau de protéine sur le gain de poids moyen quotidien (GMQ)}

Le Tableau IV montre que le traitement thermique des graines de Cajanus influence la vitesse de croissance des animaux $\left(\mathrm{P}_{\mathrm{TT}}=0,000\right)$ de même que le niveau protéique $\left(\mathrm{P}_{\mathrm{NP}}=0,000\right)$ et la combinaison des deux facteurs $\left(\mathrm{P}_{\mathrm{TT} * \mathrm{NP}}=0,018\right)$. La plus faible croissance est obtenue chez les poulets nourris avec la ration incorporant les graines séchées qui présente une différence significative avec les autres rations dès la $3^{\text {ème }}$ semaine $d^{\prime}$ 'expérimentation. Concernant le niveau de protéine, Les animaux nourris avec les rations titrées à $16 \%$ de protéines ont la plus faible croissance alors qu'aucune différence significative n'est identifiée entre le GMQ des animaux nourris avec respectivement les rations de 10 et $13 \%$ de protéine.

Tableau IV. Gain de poids moyen quotidien en fonction du traitement thermique et du niveau de protéine

\begin{tabular}{cccccccccc}
\hline $\begin{array}{c}\text { Âge } \\
\text { (semaine) }\end{array}$ & \multicolumn{3}{c}{ traitements } & \multicolumn{4}{c}{ Niveaux protéiques } & \multicolumn{3}{c}{ P value } \\
\cline { 2 - 10 } & CG & CV & CS & 10 & 13 & 16 & TT & NP & TT*NP \\
\hline 1 & $5,39^{\mathrm{b}}$ & $4,98^{\mathrm{ab}}$ & $4,80^{\mathrm{a}}$ & $5,01^{\mathrm{b}}$ & $5,37^{\mathrm{a}}$ & $4,79^{\mathrm{b}}$ & $0,000^{*}$ & $0,001^{*}$ & $0,029^{*}$ \\
& $\pm 0,10$ & $\pm 0,14$ & $\pm 0,17$ & $\pm 0,09$ & $\pm 0,11^{\mathrm{y}}$ & $\pm 0,19$ & & & \\
2 & $5,56^{\mathrm{b}}$ & $4,95^{\mathrm{ab}}$ & $4,98^{\mathrm{a}}$ & $5,23^{\mathrm{a}}$ & $5,33^{\mathrm{a}}$ & $4,93^{\mathrm{a}}$ & $0,000^{*}$ & $0,019^{*}$ & 0,263 \\
& $\pm 0,09$ & $\pm 0,10$ & $\pm 0,09$ & $\pm 0,09$ & $\pm 0,10$ & $\pm 0,11$ & & & \\
3 & $5,18^{\mathrm{b}}$ & $5,17^{\mathrm{b}}$ & $4,88^{\mathrm{a}}$ & $5,15^{\mathrm{a}}$ & $5,20^{\mathrm{a}}$ & $4,87^{\mathrm{a}}$ & $0,002^{*}$ & $0,002^{*}$ & $0,000^{*}$ \\
& $\pm 0,06$ & $\pm 0,07$ & $\pm 0,07$ & $\pm 0,06$ & $\pm 0,08$ & $\pm 0,07$ & & & \\
4 & $5,26^{\mathrm{b}}$ & $5,49^{\mathrm{b}}$ & $4,99^{\mathrm{a}}$ & $5,29^{\mathrm{a}}$ & $5,32^{\mathrm{a}}$ & $5,14^{\mathrm{a}}$ & $0,000^{*}$ & 0,245 & 0,093 \\
& $\pm 0,07$ & $\pm 0,08$ & $\pm 0,07$ & $\pm 0,06$ & $\pm 0,08$ & $\pm 0,09$ & & & \\
5 & $5,43^{\mathrm{b}}$ & $5,36^{\mathrm{b}}$ & $4,92^{\mathrm{a}}$ & $5,30^{\mathrm{ab}}$ & $5,40^{\mathrm{b}}$ & $5,02^{\mathrm{a}}$ & $0,000^{*}$ & $0,016^{*}$ & 0,886 \\
& $\pm 0,07$ & $\pm 0,08$ & $\pm 0,09$ & $\pm 0,06$ & $\pm 0,08$ & $\pm 0,10$ & & & \\
& $5,35^{\mathrm{a}}$ & $5,50^{\mathrm{a}}$ & & $5,27^{\mathrm{a}}$ & $5,40^{\mathrm{a}}$ & $5,29^{\mathrm{a}}$ & 0,195 & 0,284 & 0,167 \\
& $\pm 0,08$ & $\pm 0,08$ & & $\pm 0,07$ & $\pm 0,09$ & $\pm 0,15$ & & & \\
\hline Moyenne & $5,36^{\mathrm{b}}$ & $5,23^{\mathrm{b}}$ & $4,92^{\mathrm{a}}$ & $5,21^{\mathrm{ab}}$ & $5,32^{\mathrm{b}}$ & $4,97^{\mathrm{a}}$ & $0,000^{*}$ & $0,000^{*}$ & $0,018^{*}$ \\
& $\pm 0,05$ & $\pm 0,06$ & $\pm 0,05$ & $\pm 0,05$ & $\pm 0,05$ & $\pm 0,06$ & & & \\
\hline
\end{tabular}

Moyenne \pm écart-type

* Indique les différences significatives des effets (traitement et niveau protéique)

Les valeurs suivies de la même lettre pour une même ligne et par facteur ne sont significativement différentes au seuil de $5 \%$.

\section{Effet du traitement thermique et du niveau de protéine sur l'indice de consommation}

Les analyses statistiques révèlent une influence de traitement thermique $\left(\mathrm{P}_{\mathrm{TT}}=0,001\right)$ et du niveau protéique $\left(\mathrm{P}_{\mathrm{NP}}=0,000\right)$ sur l'indice de consommation de même qu'un effet cumulé des deux paramètres $\left(\mathrm{P}_{\mathrm{TT}} \mathrm{NP}_{\mathrm{NP}}=\right.$ 0,025) (Tableau V). Les meilleurs indices de consommation sont obtenus avec les animaux nourris avec les aliments composés de graines de Cajanus cajan torréfiées et cuites à la vapeur. Ces deux moyennes sont significativement différentes de celle des poulets nourris avec les rations à base de graines séchées. Au niveau protéique, les rations de 13 et $16 \%$ de protéine qui ne 
présentent pas de différence entre elles sont les meilleurs indices de consommation. Elles sont différentes de l'indice de consommation obtenu chez les animaux nourris avec les rations à $10 \%$ de protéine dès la 4 ème semaine.

Tableau V. Indice de consommation en fonction du traitement thermique et du niveau de protéine

\begin{tabular}{|c|c|c|c|c|c|c|c|c|c|}
\hline \multirow{2}{*}{$\begin{array}{c}\hat{\text { Âge }} \\
\text { (semaine) }\end{array}$} & \multicolumn{3}{|c|}{ Traitements } & \multicolumn{3}{|c|}{ Niveaux protéiques } & \multicolumn{3}{|c|}{ P Valeur } \\
\hline & $\mathrm{CG}$ & $\mathrm{CV}$ & $\mathrm{CS}$ & 10 & 13 & 16 & TT & NP & TT*NP \\
\hline \multirow[t]{2}{*}{1} & $3,74^{\mathrm{a}}$ & $4,65^{\mathrm{a}}$ & $4,16^{\mathrm{a}}$ & $5,46^{\mathrm{b}}$ & $3,18^{\mathrm{a}}$ & $3,92^{\mathrm{a}}$ & 0,487 & $0,007 *$ & 0,793 \\
\hline & $\pm 0,52$ & $\pm 0,55$ & $\pm 0,51$ & $\pm 0,49$ & $\pm 0,50$ & $\pm 0,57$ & & & \\
\hline \multirow[t]{2}{*}{2} & $3,75^{\mathrm{a}}$ & $5,30^{\mathrm{b}}$ & $5,35^{\mathrm{b}}$ & $5,69^{\mathrm{b}}$ & $3,76^{\mathrm{a}}$ & $4,94^{\mathrm{ab}}$ & $0,008 *$ & $0,004 *$ & 0,429 \\
\hline & $\pm 0,39$ & $\pm 0,44$ & $\pm 0,38$ & $\pm 0,37$ & $\pm 0,40$ & $\pm 0,43$ & & & \\
\hline \multirow[t]{2}{*}{3} & $4,77^{\mathrm{a}}$ & $4,87^{\mathrm{a}}$ & $5,76^{\mathrm{a}}$ & $5,21^{\mathrm{a}}$ & $4,27^{\mathrm{a}}$ & $5,94^{\mathrm{a}}$ & 0,319 & 0,101 & 0,550 \\
\hline & $\pm 0,51$ & $\pm 0,56$ & $\pm 0,49$ & $\pm 0,56$ & $\pm 0,52$ & $\pm 0,48$ & & & \\
\hline \multirow[t]{2}{*}{4} & $4,19^{\mathrm{a}}$ & $4,88^{a}$ & $5,77^{\mathrm{a}}$ & $7,28^{b}$ & $4,28^{\mathrm{a}}$ & $3,27^{\mathrm{a}}$ & $0,008 *$ & $0,000 *$ & $0,001 *$ \\
\hline & $\pm 0,34$ & $\pm 0,35$ & $\pm 0,34$ & $\pm 0,30$ & $\pm 0,35$ & $\pm 0,38$ & & & \\
\hline \multirow[t]{2}{*}{5} & $5,20^{\mathrm{a}}$ & $5,32^{\mathrm{a}}$ & $7,75^{\mathrm{b}}$ & $8,41^{\mathrm{b}}$ & $5,31^{\mathrm{a}}$ & $4,55^{\mathrm{a}}$ & $0,001 *$ & $0,000 *$ & 0,056 \\
\hline & $\pm 0,44$ & $\pm 0,45$ & $\pm 0,54$ & $\pm 0,36$ & $\pm 0,45$ & $\pm 0,60$ & & & \\
\hline \multirow[t]{2}{*}{6} & $6,17^{\mathrm{a}}$ & $5,60^{\mathrm{a}}$ & & $9,46^{\mathrm{b}}$ & $5,78^{\mathrm{a}}$ & $4,32^{\mathrm{a}}$ & 0,260 & $0,000 *$ & $0,003 *$ \\
\hline & $\pm 0,35$ & $\pm 0,36$ & & $\pm 0,29$ & $\pm 0,40$ & $\pm 0,91$ & & & \\
\hline \multirow[t]{2}{*}{ Moyenne } & $4,62^{\mathrm{a}}$ & $4,98^{\mathrm{a}}$ & $5,95^{\mathrm{b}}$ & $6,89^{b}$ & $4,16^{\mathrm{a}}$ & $4,50^{\mathrm{a}}$ & $0,001 *$ & $0,000 *$ & $0,025^{*}$ \\
\hline & $\pm 0,25$ & $\pm 0,27$ & $\pm 0,25$ & $\pm 0,24$ & $\pm 0,25$ & $\pm 0,28$ & & & \\
\hline
\end{tabular}

Moyenne \pm écart-type

*indique les différences significatives des effets (traitement et niveau protéique)

Les valeurs suivies de la même lettre pour une même ligne et par facteur ne sont significativement différentes au seuil de $5 \%$.

Effet du traitement thermique et de la teneur en protéine sur la digestibilité de la protéine

Le coefficient de digestibilité apparent présente une différence significative entre les rations constituées de graines séchées et celles cuites à la vapeur pour tous les paramètres (MS, MO, protéines et cendres brutes) comme indiqué dans le Tableau VI. Pour le niveau protéique, il est noté également une différence significative entre les différents niveaux pour la matière sèche et la matière organique. Pour la protéine, la différence significative se situe entre le niveau $13 \%$ et $16 \%$ alors qu'ils ne sont pas différents pour les cendres brutes. Les résultats du Tableau VI montrent que la nature du traitement et le niveau protéique ont significativement influencé la digestibilité des aliments. Les animaux ont mieux digéré les aliments formulés avec les graines de Cajanus cajan torréfiées et celles cuites à la vapeur. Une baisse de la digestibilité est observée avec les aliments à $16 \%$ pour la matière sèche, la matière organique et les protéines brutes. 
Tableau I. Effet du traitement thermique et du taux de protéine sur la digestibilité de la protéine

\begin{tabular}{ccccrrrrrr}
\hline \multirow{2}{*}{ Paramètres } & \multicolumn{3}{c}{ Traitements } & \multicolumn{4}{c}{ Niveaux protéiques } & \multicolumn{3}{c}{ P Valeur } \\
\cline { 2 - 10 } & CG & CV & CS & 10 & 13 & 16 & TT & NP & TT*NP \\
\hline CUDa MS & $88,09 \mathrm{ab}$ & $90,45 \mathrm{~b}$ & $85,92 \mathrm{a}$ & $91,87 \mathrm{c}$ & $88,64 \mathrm{~b}$ & $83,94 \mathrm{a}$ & $0,000^{*}$ & $0,000^{*}$ & $0,000^{*}$ \\
& 4,51 & 1,60 & 5,13 & 1,36 & 2,62 & 4,20 & & & \\
CUDa MO & $88,22 \mathrm{ab}$ & $90,48 \mathrm{~b}$ & $86,05 \mathrm{a}$ & $92,10 \mathrm{c}$ & $88,74 \mathrm{~b}$ & $83,91 \mathrm{a}$ & $0,000^{*}$ & $0,000^{*}$ & $0,000^{*}$ \\
& 4,56 & 1,66 & 5,30 & 1,30 & 2,51 & 4,26 & & & \\
CUDa & $78,73 \mathrm{ab}$ & $80,44 \mathrm{~b}$ & $75,93 \mathrm{a}$ & $80,05 \mathrm{a}$ & $80,07 \mathrm{a}$ & $74,97 \mathrm{~b}$ & $0,000^{*}$ & $0,000^{*}$ & 0,45 \\
protéine & 3,28 & 3,29 & 3,90 & 2,90 & 3,34 & 3,14 & & & \\
CUDa & $81,08 \mathrm{ab}$ & $87,85 \mathrm{~b}$ & $77,79 \mathrm{a}$ & $77,64 \mathrm{a}$ & $84,09 \mathrm{~b}$ & $84,99 \mathrm{~b}$ & $0,000^{*}$ & $0,000^{*}$ & $0,000^{*}$ \\
Cendre & 1,87 & 7,00 & 7,43 & 5,58 & 9,33 & 3,35 & & & \\
\hline
\end{tabular}

Moyenne \pm écart-type

*indique les différences significatives des effets (traitement et niveau protéique)

Les valeurs suivies de la même lettre pour une même ligne et par facteur ne sont significativement différentes au seuil de $5 \%$.

\section{Effet de l'ingestion des aliments sur le taux de mortalité}

Le Tableau VIII indique les taux de mortalité déterminés au cours de l'étude. Des mortalités ont été constatées à partir de la 4ème semaine au niveau des lots nourris avec les graines torréfiées et cuites à la vapeur pour les rations de $16 \%$ de protéine. Pour les animaux nourris avec graines séchées, c'est avec les rations 13 et $16 \%$ de protéine que des mortalités ont été enrégistrées. La mortalité est nulle pour le reste. Elle est plus faible pour la ration à base de graines cuites à la vapeur de niveau $16 \%$ de protéines (CV47).

Tableau VIII. Taux de mortalité cumulé des poulets en fonction du traitement thermique et du niveau protéique

\begin{tabular}{|c|c|c|c|c|c|c|c|c|c|}
\hline \multirow{4}{*}{ Paramètres } & \multicolumn{9}{|c|}{ Traitements thermiques des graines de Cajanus cajan } \\
\hline & \multicolumn{3}{|c|}{ Torréfaction } & \multirow{2}{*}{\multicolumn{3}{|c|}{$\begin{array}{l}\text { Cuisson à la vapeur } \\
\text { Taux de protéines (\%) }\end{array}$}} & \multicolumn{3}{|c|}{ Séchage } \\
\hline & & & & & & & & & \\
\hline & 10 & 13 & 16 & $\mathbf{1 0}$ & 13 & 16 & 10 & 13 & 16 \\
\hline \multicolumn{10}{|l|}{ Semaine 1} \\
\hline \multirow{2}{*}{\multicolumn{10}{|c|}{$\begin{array}{l}\text { Semaine } 2 \\
\text { Semaine } 3\end{array}$}} \\
\hline & & & & & & & & & \\
\hline Semaine 4 & & & 33,33 & & & & & 28,57 & \\
\hline Semaine 5 & & & 50,00 & & & 20,00 & & 42,86 & 66.67 \\
\hline Semaine 6 & & & & & & & & 57,14 & 100 \\
\hline Total & & & $\mathbf{5 0 , 0 0}$ & & & 20,00 & & 57,14 & 100 \\
\hline
\end{tabular}

\section{Discussion}

Les résultats montrent que la prise d'aliment est influencée par le niveau protéique et diminue avec l'augmentation du niveau protéique. En effet, une augmentation du niveau protéique correspond à une augmentation du taux d'incorporation de graines de Cajanus cajan dans la ration d'environ 11,32 et $48 \%$ respectivement pour les niveaux 10, 13 et 16. Le même constat a été fait par Ani et Okeke (2003) qui ont montré que l'incorporation à plus de 
$27 \%$ de pois d'angole avait un effet dépressif sur la consommation alimentaire chez le poulet de chair. Des observations similaires ont été faites par d'autres auteurs utilisant d'autres légumineuses à graines comme source de protéine. C'est le cas de Mukhtar (2007) lors de ses travaux sur le poulet de chair nourri avec des graines de Bissap (Hibiscus sabdariffa) incorporées entre 0 et $20 \%$ dans la ration. Des effets du traitement thermique appliqué aux graines sur la consommation alimentaire ont également été déterminés. Les rations à base de graines torréfiées ou cuites à la vapeur ont enregistrées les plus faibles consommations. Amaefule et Obioha (2005) abordent dans le même sens et affirment que pour des taux d'incorporation inférieurs ou égaux à $10 \%$, les traitements n'influencent pas la prise alimentaire chez le poulet or, nos taux d'incorporation sont supérieurs à $10 \%$.

Concernant le gain de poids moyen quotidien, le traitement thermique a eu un effet significatif tout le long de ce travail. Les animaux nourris avec les aliments à base de graines de Cajanus cajan séchées ont obtenu les gains de poids les plus faibles alors que ceux ayant reçu les aliments à base de graines de Cajanus cajan cuites à la vapeur et torréfiées ont acquis de meilleur gain de poids. Nos résultats corroborent ceux de Amaefule et Obioha (2005) qui ont trouvé que le gain pondéral variait selon le traitement thermique. Cette différence pourrait être attribuée à l'action du traitement thermique sur des substances anti-nutritionnelles contenues dans les graines de Cajanus cajan. Ces facteurs auraient une influence sur la digestibilité et l'assimilation des nutriments. En effet, Udensi et al. (2007) ont montré pour le pois chiche que la torréfaction et la cuisson à la vapeur réduisaient la teneur des graines en facteurs anti-trypsiques. Egounlety et Aworh (2003) ont aussi montré l'effet de la cuisson pendant 15 à 30 min sur leur composition en facteurs antitrypsiques des graines de soja, de niébé et féverole avec une réduction de 76 à $86 \%$. De plus, Chrysostome et al. (1998) ont montré que si la cuisson n'a pas d'effet sur la teneur en protéine, elle améliore l'énergie métabolisable ce qui a un effet positif sur la prise de poids. L'adjonction de graines de Cajanus cajan à différents taux d'incorporation (différents niveaux de protéine) a significativement influencé $(\mathrm{p}<0,005)$ le gain de poids moyen quotidien. Les animaux nourris aux aliments $13 \%$ ont eu un gain de poids supérieur à ceux nourris aux aliments $10 \%$ peut-être à cause du taux plus élevé en protéine. La baisse de poids observée chez les animaux nourris avec les aliments titrés à 16 $\%$ s'expliquerait par le niveau d'incorporation de graines plus élevé (47,95\%) qui entraine une plus grande ingestion de facteurs antinutritionnels dont les tanins qui déséquilibreraient l'absorption des nutriments (Hamad, 2000). L'augmentation du taux de protéine a présenté un effet positif sur l'indice de consommation.

L'indice de consommation est plus élevé chez les poussins nourris aux aliments contenant $10 \%$ de protéine et nettement meilleur chez ceux nourris 
avec les aliments à 13 et $16 \%$. Cela est dû à la plus forte consommation alimentaire des rations à $10 \%$ de protéines alors le gain de poids est statistiquement identique entre les animaux nourris avec 10 et $13 \%$. Ainsi, en petite quantité les rations de $13 \%$ semblent satisfaire les besoins de l'animal.

Les animaux ont mieux digéré les aliments formulés avec les graines de Cajanus cajan torréfiées et celles cuites à la vapeur $(81,99 \%$ et 81,70\%). Cela pourrait être dû à l'élimination du facteur antitrypsine par ces traitements, ce qui améliore la digestibilité. En effet, Hendricks et Bailey (1989) affirment que les facteurs antinutritionnels abaissent le coefficient de transformation de l'aliment quand leurs concentrations sont élevées ce qui pourrait être le cas pour les graines séchées.

Au niveau du taux de mortalité, il augmente avec le taux de protéine de l'aliment et est également influencé par le traitement thermique. Le taux de mortalité est plus important chez les sujets consommant des rations à $16 \%$ de protéine que pour les autres aliments. Cela est lié au taux d'incorporation qui est plus important avec probablement une ingestion plus importante de facteurs antinutritionnels qui selon Hendricks et Bailey (1989) provoquent l'anorexie et des retards de croissance. On comprend que les aliments avec un taux d'incorporation élevé auront une concentration supérieure, donc un effet plus marqué. Le faible taux de mortalité enregistré avec des rations à base de graines cuites à la vapeur ou torréfiées s'expliquerait par l'inactivation d'une grande proportion de ces facteurs dûs à l'effet de ces deux traitements. Cela expliquerait également la mortalité élevée chez les poulets nourris avec les graines séchées au niveau des rations enrichies en protéines à 13 et $16 \%$. Ces résultats s'alignent sur celui de Grimaud (1991) qui a constaté une mortalité élevée avec une incorporation de graines non traitées de Cajanus cajan supérieure à $25 \%$ en complément d'un aliment commercial. Il a lié ce fait à la présence de facteurs antinutritionnels dans la graine de Cajanus cajan puisqu'il a pu mettre en évidence un facteur antitrypsine.

\section{Conclusion}

L'impact simultané de trois taux de protéines $(10 \%, 13 \%$ et $16 \%)$ et trois types de traitement thermique (torréfaction, cuisson à la vapeur et séchage) a été évalué à travers les performances zootechniques des poussins d'un jour nourris durant 6 semaines. Il ressort de ce travail que les poussins extériorisent mieux les performances de croissance avec les aliments à $13 \%$ de protéine (taux d'incorporation 32\%) et dont les graines de Cajanus ont été torréfiées ou cuites à la vapeur. Ces aliments ont induit un gain de poids supérieur avec un meilleur indice de consommation et un taux de mortalité nul. Cajanus cajan apparaît donc comme une matière première locale intéressante pour l'alimentation des poulets locaux. Le taux d'incorporation peut être améliorer par le traitement à la vapeur vue qu'au taux d'incorporation 
de $48 \%$ les mortalités restent faibles. Il faudra toutefois évaluer l'effet du temps de cuisson sur la réduction des facteurs antinutritionnels.

\section{References:}

1. Aklobessi, K., Gitoba, K., Kokou, G., \& Kougbenyal, L. (1992). Evaluation de la méthodologie d'étude de base de la population avicole rurale en Afrique (Rapport Togo). Dakar : C.R.D.I, bureau régional pour l'Afrique centrale et occidentale, 20p.

2. Akouango, F., Mouangou, J. \& Ganougo, G. (2004). Phénotypes et performances d'élevage chez des populations locales de volailles du genre "Gallus gallus" au Congo Brazzaville. Cahiers Agricultures, volume 13, pp. 257-263.

3. Alla-Sombo, E. \& Fousseni, N. (2005). Céchi, cet eldorado oublié du développement In Reseauivoire.net [En ligne]. < http://www.rezoivoire.net/ivoire/villes-villages/422/cechi-ceteldorado-oublie-du-developpement.html>, consulté le 11 mai 2018.

4. Amaefule, K. \& Obioha, F. (2005). Performance of pullet chicks fed raw or processed pigeon pea (Cajanus cajan) seed meal diets. Livestock Research for Rural Development [En ligne]. Volume 17, n³. Récupéré sur « http://www.Irrd.org/lrrd17/3/amae17029.htm »

5. Ani, A. \& Okeke, G. (2003). La substitution de la farine de soja par le pois d'angole (Cajanus cajan) dans l'alimentation des poulets de chair In NSAP, Acte de la 28ème conférence. Ibadan, pp 10-12.

6. Ayssiwede, S.B., Missoko-Mabeki, R., Mankor, A., Dieng, A., Houinato, M.R., Chrysostome, C.A.A.M., Dahouda, M., Missohou, A., \& Hornick, J.L. (2012). Effets de l'incorporation de la farine de feuilles de Cassia tora (Linn.) dans la ration alimentaire de jeunes poulets traditionnels du Sénégal. Revue Médecine Vétérinaire., 2012, 163, 8-9, 375-386.

7. Chrysostome, C., Xu Bing, An., Bonou, M., \& Delpech, P. (1998). Variation de l'énergie métabolisable du pois d'Angole (Cajanus cajan) cru et cuit chez le poulet et la pintade. Revue Élevage et de Médecine. Vétérinaire des Pays Tropicaux, 51 (2) : 131-133.

8. Egounlety, M. \& Aworh, O. C. (2003). Effet du trempage, du décorticage, de la cuisson et de la fermentation avec Rhizopus oligosporus sur les oligosaccharides, l'inhibiteur de la trypsine, l'acide phytique et les tanins de soja (Glycine max Merr.), de niébé (Vigna unguiculata L. Walp) et de féverole (Macrotyloma geocarpa Harms). Journal de génie alimentaire, Volume 56, numéros 2-3, février 2003, pages 249-254. 
9. FAO (2004). Production en aviculture familiale : un manuel technique, par Sonaiya E. B. et Swan S. E. J. Manuel de production et santé animales FAO, $\mathrm{N}^{\circ} 1$. Rome, Italie. 140 p.

10. Grimaud, P. (1991). Incorporation de matières premières locales dans des régimes alimentaires pour porcs et volailles. Fiche technique. Institut d'Elevage et Médecine Vétérinaire des pays Tropicaux. Païta - nouvelle calédonie, $11 \mathrm{p}$.

11. Grimaud, P. (1988). The pigeon pea (Cajanus cajan) : a possible alternative for traditional pig and poultry farming in New Caledonia. Revue d'Elevage et de Médecine Vétérinaire de Nouvelle-Calédonie, 11: 29-36.

12. Guèye, E. F. (1998). Village egg and fowl meat production in Africa. World's Poultry Science Journal, volume 54, n²1, pp. 73-86.

13. Gueye, E. F. (2000). The role of family poultry in poverty alleviation, food security and the promotion of gender equality in rural Africa. Outlook on Agriculture, 2000, 29, 129-136.

14. Hamad, B. (2000). Effect of feeding corticated and decorticated $(C$. Cajan) seeds on broiler chicks performance. Animal Production Thesis. University of Khartoum.

15. Hendricks, J. D. \& Bailey, G. S. (1989). Des toxines adventices. Dans JE Halver, éd. Nutrition du poisson. Presse académique. New York, p. 605-651.

16. Missohou, A., Dieye, P. \& Talaki, E. (2002). Rural poultry production and productivity in southern Senegal. Livestock Research for Rural Developpement, volume $14, \quad \mathrm{n}^{\circ} 2, \quad 6 \mathrm{p}$. Récupéré sur « http://www.lrrd.cipav.org.co/lrrd14/2/miss142.htm »

17. Mukhtar, A. M. (2007). The Effect of feeding rosella (Hibiscus sabdariffa) seed on broiler chick's performance. Research Journal of Animal and Veterinary Science, $2: 21-23$.

18. Niyonkuru, D. (2002). La culture du pois cajan : un trésor méconnu en Afrique centrale. Éditions SAILD, collection expériences des fédérations no 10, Cameroun, 23p.

19. Okandza, Y., Mopoundza, P., Dimi Ngatse, S., Halbouche, M. \& Akouango, P. (2017). Influence de la substitution graduelle de tourteau de soja par la féverole sur la croissance et la conformation de la carcasse chez les poulets de chair. Journal of Applied Biosciences, 110: 10714-10720.

20. Ouattara, S., Bougouma-Yameogo, V.M.C., Nianogo, A.J. \& Al Bachir, A. (2014). Effets des graines torréfiées de Vigna unguiculata (niébé) comme source de protéines, dans l'alimentation des poules locales en ponte au Burkina Faso, sur leurs performances 
zootechniques et la rentabilité économique des régimes. International Journal of Biological Chemical Sciences 8(5): 1990-1999.

21. Ouedraogo, B., Bayala, B., Zoundi, J. S., \& Sawadogo, L. (2015). Effet de l'incorporation de graines d'Hibiscus Sabdariffa (Oseille de Guinée) dans l'alimentation sur quelques paramètres d'ingestion et de croissance du poulet en aviculture traditionnelle améliorée au Burkina Faso. Agronomie Africaine 27 (3) : 269 - 283.

22. Ouedraogo, M. (2001). Contribution à l'étude de l'impact de la variabilité climatique sur les ressources en eau en Afrique de l'ouest. Analyses des conséquences d'une secheresse persistante: Nomes hydrologiques et modelisation régionale. Thèse de doctorat ès Geosciences, Université Montpellier II, France, 258 p.

23. Picq, J. J., Delmont, J. \& Mosny, Y. (1992). Zones bioclimatiques tropicales et pathologie. Medecine d'Afrique noire, 39 (3), p 157-161.

24. Saliou, K. \& Danho, T. (2008). Côte d'Ivoire: Revue du secteur avicole. FAO, Division de la production et de la santé animales, centre d'urgence pour les maladies animales transfrontalières, Unité de socioéconomie, production et biodiversité Côte d'Ivoire. Rome, Italie. 77 p.

25. Sarkar, K. \& Bell, J. (2006). Potentiel du poulet indigène et son rôle dans la lutte contre la pauvreté et dans la sécurité alimentaire pour les ménages ruraux. Rapport de recherche, $\mathrm{n}^{\circ} 3$. Bulletin RIDAF, volume $16, \mathrm{n}^{\circ} 2$, pp. 16-28.

26. Sonaiya, E. B. (1995). Feed ressources for smallholder rural poultry in Nigeria. World Animal Review, volume 82, n 1 , pp.25-33.

27. Udensi, E A., Ekwu, F C., \& Isinguzo, J. N. (2007). Antinutrient factors of vegetable cowpea (Sesquipedallis) seeds during thermal processing. Pakistan Journal of Nutrition, 6 (2): 194-197. 